Cases of this type in which the cyst is the entire detachment have previously been described by Weve and Butler. Other types of retinal cysts may be summarised.

A type of cyst associated with true retinal detachment has been described by Weve and Cridland. Here the cyst is probably subretinal, the surrounding retina being raised from the choroid presumably by the growing cyst. A retinal tear is usually present in addition. Only one such case has been seen by the writer; it occurred in a girl, aged 13 years, and the cyst had ruptured.

In passing, other cysts of the retina may be mentioned. Small congenital cysts have been described by McCulloch arising from the optic disc, and by Stieren in opaque nerve fibres; degenerative cysts from subretinal haemorrhage in Lindau's disease and in probable Coats' disease by Hine, small cysts at the macula, often bilateral, and associated with great disturbance of central vision. Finally, there are the small cysts found in the anterior portions of the retina of almost all old people, which seem to be of little clinical significance.

My thanks are due to the staff of Moorfields, and, in particular, to Mr. Goulden and Mr. Whiting, for the material on which this paper is based.

\title{
REFERENCES
}

Weve.-Arch.f. Augenheilk., Vol. CIX, 1935.

Cridland.-Trans. Ophthal. Soc. U.K., Vol. XLIV, 1924.

Butler.-Trans. Ophthal. Soc. U.K., Vol. XLII, 1922.

Stieren.-Trans. Amer. Ophthal. Soc., Vol. XXXI, 1933.

McCulloch.-Trans. Ophthal Soc. U.K., Vol. L, 1930.

Hine.-Trans. Ophthal. Soc. U.K., Vol. XLVIII, 1928.

Samuels.-Trans. Amer. Ophthal. Soc., Vol. XXVIII, 1930.

\section{THE TREATMENT AND COMPLICATIONS OF CHALAZIA}

BY

Allan H. Briggs

HOUSE SURGEON, ROYAL LONDON OPHTHALMIC HOSPITAL

THE chalazion, or Meibomian granuloma, is so much a commonplace of ophthalmic practice that it stands in some danger of being treated with an unmerited contempt, born of long familiarity. In standard text-books the treatment is usually dismissed in a few lines; de minimis non curat lex; but in actual practice the traditional operation of incision and curettage is not so invariably satisfactory as one is led to suppose. Admittedly, most cases are benefited, and the majority cured, by this simple procedure; but 
there remains a large number of old-standing or complicated cases which are not cured by this operation, even when it has been repeatedly performed; these folk suffer much inconvenience and annoyance, and it is with them that we are especially concerned. To effect a permanent and satisfactory cure requires more radical treatment ; the reason for this lies in the pathology of the condition.

Pathology.-Acute inflammation of a Meibomian gland is relatively uncommon. It may arise in a previously healthy gland, or as a secondary pyogenic infection of a pre-existing chalazion. In the former case, suppuration usually takes place rapidly, and pus discharges from the pouting orifice of the duct of the affected gland. The contents can easily be expressed through the duct, and resolution takes place very rapidly. Secondarily infected chalazia often show an obvious source of pyogenic infection, usually a stye in close proximity on the same lid, or on the other lid at the point of contact. Their treatment will be discussed later.

The chalazion is a chronic inflammation of a Meibomian gland, of unknown origin. It is usually attributed to a low-grade infection by an organism of low virulence. There is a desquamative catarrh of the affected gland, with some periadenitis. A small solid swelling gradually develops, consisting of granulation tissue. This granuloma gradually increases in size, its presence in the lid giving rise to a variable amount of fibrosis, so that in the course of several weeks or months, if untreated, it may become surrounded by what is virtually a capsule of fibrous tissue, from which fibrous septa may be extended into the interior of the mass. The centre of the granuloma sooner or later breaks down into necrotic débris. These two processes-central necrosis and peripheral fibrosisoccur side by side, the extent of each change in different cases varying very much. There is thus a series of possible end-results; at one end of the series is the traditional "Meibomian cyst," a thin-walled fibrous sac filled with greenish glairy sterile fluid, comparable to a cold abscess; while at the other end of the series, if fibrosis has been the predominating factor, the result is a semisolid or solid fibrotic nodule. This nodule has a more or less well defined capsule, and consists of interlacing fibrous septa in which globules of the glairy fluid may be held much as globules of fluid are contained in the fibrous framework of an orange. Any intermediate stage between these extremes may be encountered.

To this basic condition complications are frequently added.

The infection may spread along the duct of the affected gland, and result in a small granuloma of the lid margin; or it may erode through the superficial layers of the tarsal plate, especially in the upper lid, forming a swelling beneath the skin, which becomes greatly stretched and thinned over it. Secondary infection then frequently occurs, and the skin breaks down, with the formation 
of a sinus. A more common complication is rupture into the conjunctival sac, again with the formation of a sinus, from the mouth of which masses of granulation tissue may sprout.

Treatment.- In the uncomplicated case, the first step is to make an incision into the chalazion from the conjunctival aspect. The incision is made at right angles to the lid margin, throughout the whole length of the swelling. Only in the rarest instances is it necessary, or justifiable, to make an incision from the skin surface of the lid. Transverse or cruciate incisions should never be employed ; such wounds heal but slowly, with much fibrosis and distortion of the conjunctival aspect of the lid; and it is possible that they may result in the development of further chalazia from the infection of neighbouring glands whose natural drainage is cut off in the subsequent fibrosis.

Anaesthesia is best achieved by infiltrating the lid with four per cent. novocaine ; if the injection is carried well up to the lid margin, the operation is quite painless. Cocaine drops may be instilled as well, but this is usually unnecessary, and it is perhaps better not to have the cornea anaesthetised; a corneal abrasion can easily be unwittingly inflicted on a restless patient. If solid cocaine is rubbed on to the conjunctiva (the usual custom), and novocaine is not employed, only the conjunctiva is anaesthetised; the deeper parts of the lid are still sensitive, and pain is always felt. Solid cocaine is better, however, for small children, who are usually terrified by a subcutaneous injection.

Whether a chalazion clamp' is employed is a matter of personal taste; it is usually rather resented by the patient, but it is a great convenience when operating single-handed, and offers the advantage of a bloodless field of operation. The site of election for the incision is usually shown by a greyish or yellowish discolouration of the swelling; failing this, the most prominent part of the swelling is chosen. The use of a clamp renders small chalazia much more prominent.

For descriptive purposes, once the incision has been made, one of four conditions may be revealed.

(1) Frank pus is evacuated, if the chalazion has become secondarily infected. An abscess cavity remains. If curetting is attempted, it should be done very gently, for fear of spreading the infection.

(2) The traditional Meibomian "cyst"-a thin-walled sac is found, containing thin green fluid, or glairy mucoid material, corresponding to a cold abscess. The contents are easily curetted away, and these cases heal up rapidly and rarely, if ever, give rise to further trouble.

(3) In some long-standing cases, which are far from uncommon, the contents of the swelling are more or less fibrotic, with fluid 
interspaces resembling those of an orange. Curetting accomplishes little or nothing ; the curette, so to speak, bounces off the swelling.

(4) In some rare cases the swelling is found to be quite solid and fibrotic, with a more or less well-defined fibrous capsule.

Some of the cases in this group are probably not chalazia at all, but adenomata of the Meibomian glands, or even (rarely) gummata ; but microscopic section shows that some are true solid fibrogranulomata.

The treatment for these last two types of case is the same. Incision and curettage is not enough, even when repeated; a fibrotic mass remains in the lid, and each subsequent operation achieves nothing and merely adds to the fibrosis. A satisfactory and permanent cure can only be obtained by dissection and removal of the whole mass. This is not as formidable an undertaking as one might expect ; the usual incision is quite sufficient ; it is carried down until the chalazion has been divided into two parts, and these can then be shelled out with comparative ease. The subsequent haemorrhage is not, as a rule, much greater than after a vigorous curetting.

After-treatment.-A firm pad is applied to the eye when the operation is completed, and the patient sits upright and holds the pad firmly to the eye for several minutes. When all the bleeding has ceased, the pad is removed and the eye is irrigated with some bland lotion. If pus was evacuated from the chalazion, it is, perhaps, as well to instil protargol drops; otherwise parolene is instilled as a lubricant. The eye is then bandaged for 24 hours. The bandage can be dispensed with next day, and the eye is bathed twice daily with some simple lotion. Healing is complete in about a week.

Hot bathing is unnecessary, except perhaps in very infected cases ; it is undesirable, since it tends, if anything, to encourage bleeding.

\section{Complications}

Operative.-If the duct is involved, or there is a granuloma of the lid margin, there is always a granuloma of the corresponding gland as well, which must be treated along the usual lines. At the same time the granuloma of the lid margin must be excised or snipped off with scissors, and the duct laid open and curetted. If the incision into the chalazion itself is made in the usual way (that is, towards the lid margin), it is not easy to bring the knife accurately along the corresponding duct ; in such cases, therefore, it is easier to make the incision backwards, so to speak, by inserting the point of the knife into the orifice of the duct, which can usually easily be recognised, and completing the incision by cutting away.

from the lid margin. 
If the chalazion has extended through the tarsal plate and formed a swelling beneath the skin, it is usually inadvisable to incise it from the skin surface, however tempting it may be to do so. The customary incision should be made, and carried right through the tarsal plate, as in opening a collar-stud abscess. If the chalazion is of the fibrotic type, it should be dissected out; this may entail removing a small portion of the tarsal plate as well; no harm results from this procedure, since fibrous tissue is laid down when the incision heals; the gap in the tarsal plate is filled in, and no deformity at all remains. If the dissection has been incomplete, and a fibrotic mass has been left behind beneath the skin, this may be excised later through a skin incision; it is advisable to wait until all inflammation has subsided before this is attempted; these cases are very liable to secondary infection.

These chronic chalazia often grow to a very large size, and lose their spherical shape, becoming multilocular and apparently growing from multiple centres. They ramify in the tissues of the lid in all directions. Careful dissection offers the only hope of a permanent cure with a good cosmetic result, radical though the procedure may seem to be.

If the chalazion has already ruptured into the conjunctival sac, a mass of granulation tissue usually sprouts from the opening. This should first be curetted away, or snipped off with scissors; the chalazion is then incised and treated in the usual way, the incision being directed so as to pass through the sinus.

At the time of operation it is not infrequently found that many of the other Meibomian glands are infected to some extent; mucus or mucopus may be expressed from the orifices of the ducts. The sepsis may be sufficient to justify the term "pyorrhoea of the lid margin." It seems reasonable to suppose that such cases will benefit by the performance of "Meibomian massage" at intervals for a few weeks; that is to say, by inserting a glass rod under the lid, and milking the contents of the infected glands towards the lid margin, counterpressure being exerted on the outside of the lid by the finger.

Post-operative.-Severe haemorrhage is rare. Occasionally a small arterial twig is divided in the course of the operation, and bleeds when the pressure of the chalazion clamp is released; this can easily be stopped by grasping the bleeding point with a pair of small artery forceps, and crushing it. We have only encountered two cases of free haemorrhage; one, a lady of 45 years of age, who had a history of copious haemorrhage at previous minor operations; and the other a girl, aged 15 years, who had a secondary haemorrhage on the seventh day following her operation.

In both cases the haemorrhage was easily controlled by suturing the conjunctiva. 


\section{Summary}

Discussion of the common complications and pathology of the chalazion.

The customary operation of incision and curettage is not sufficient to effect a satisfactory permanent cure in long-standing or complicated cases; dissection and removal under novocaine infiltration anaesthesia is to be preferred.

Discussion of appropriate treatment for the various complications.

The author wishes to acknowledge his indebtedness to the honorary staff of Moorfields Eye Hospital for the material from which this paper has been prepared, and for their unfailing kindness and generous assistance at all times; and also to Mr. Law, pathologist to the hospital, for his encouragement and advice.

\section{EXPERIENCES OF A SUFFERER FROM WORD-BLINDNESS}

The BRIT. JL. OF OpHTHAL. has received the following note on the experiences of a patient suffering from wordblindness.

I HAVE been asked to write my experiences of word-blindness. I am not sure that I shall be able to convey the daily difficulties which this condition causes, partly because one unconsciously expects others to understand without explanation and also partly owing to the sufferer getting more or less used to the condition and consequently failing to analyse it.

My father and his only brother were both stammerers, their three sisters being normal. I had two brothers and a sister older than myself and a sister and brother younger than I. The governess who started us " took no trouble over me," "was bored with me because I was so slow," she told me this some 45 years later when I happened to meet her after that interval. Meanwhile, she had had a boys' school. She also volunteered that she knew now that she ought to have taken all the more trouble. "Reading without tears" was used for me, but without either of the desired results. My brothers and sisters went ahead-while I never got properly started. A German governess took her place when I was 7 years of age. She tried punishing me for being lazy and inattentive-which I am sure I was, for I found reading was so 